\title{
Lymph node tissue kallikrein-related peptidase 6 mRNA: a progression marker for colorectal cancer
}

\author{
L Ohlsson', G Lindmark², A Israelsson', R Palmqvist ${ }^{3}$ Å Öberg ${ }^{4}$, M-L Hammarström' and S Hammarström*,I \\ 'Department of Clinical Microbiology, Immunology, Umeå University, SE-90185 Umeå, Sweden; ${ }^{2}$ Department of Surgery, Helsingborg Hospital/Lund \\ University, SE-25 187 Helsingborg, Sweden; ${ }^{3}$ Department of Medical Biosciences, Pathology, Umeå University, Umeå SE-90 I 85, Sweden; ${ }^{4}$ Department of \\ Surgery and Perioperative Sciences, Surgery, Umeå University, SE-90185 Umeå, Sweden
}

BACKGROUND: A most important characteristic feature for poor prognosis in colorectal cancer (CRC) is the presence of lymph node metastasis. Determination of carcinoembryonic antigen (CEA) mRNA levels in lymph nodes has proven powerful for quantification of disseminated tumour cells. Here, we investigate the utility of human tissue kallikrein-related peptidase 6 (KLK6) mRNA as a progression biomarker to complement CEA mRNA, for improved selection of patients in need of adjuvant therapy and intensified follow-up after surgery.

METHODS: Lymph nodes of PTNM stage I-IV CRC- (I 66 patients/503 lymph nodes) and control (23/I 08) patients were collected at surgery and analysed by quantitative RT-PCR.

RESULTS: Lymph node KLK6 positivity was an indicator of poor outcome (hazard ratio 3.7). Risk of recurrence and cancer death increased with KLK6 lymph node levels. Patients with KLK6 lymph node levels above the 90th percentile had a hazard ratio of 6.5 and 76 months shorter average survival time compared to patients with KLK6 negative nodes. The KLK6 positivity in lymph nodes with few tumour cells, that is, low CEA mRNA levels, also indicated poor prognosis (hazard ratio 2.8).

CONCLUSION: In CRC patients, lymph node KLK6 positivity indicated presence of aggressive tumour cells associated with poor prognosis and high risk of tumour recurrence.

British Journal of Cancer (2012) I 07, I50-157. doi:I0.1038/bjc.20 I2.220 www.bjcancer.com

Published online 14 June 2012

(C) 2012 Cancer Research UK

Keywords: colorectal neoplasms; lymphatic metastasis; neoplasm staging; prognosis; biomarker mRNA; quantitative RT-PCR

The most important prognostic characteristic of colorectal cancer (CRC) patients resected for cure is presence or absence of lymph node metastasis (Chang et al, 2007; Iddings and Bilchik, 2007; Nicastri et al, 2007). Approximately $50 \%$ of patients with tumour positive lymph nodes, that is, stage III CRC (anyTN1-2M0) and about $25 \%$ of the patients apparently without lymph node involvement, that is, stage I (T1-2N0M0) and II (T3-4N0M0) patients will recur (Bilchik et al, 2007; Iddings and Bilchik, 2007; Nicastri et al, 2007). Therefore, it is of utmost importance to detect tumour cells in lymph nodes, and to determine their metastatic potential. Thus, undertreatment of stage I and II patients as well as overtreatment of stage III patients may be avoided, improving tailored adjuvant treatment and follow-up.

A significant number of stage I and II patients, who will experience tumour recurrence probably harbour occult tumour cells in their lymph nodes. This is most likely due to the inadequate sensitivity of routine histopathological staining procedure and to the insufficient number of examined lymph nodes (Nicastri et al, 2007). Biomarkers, like carcinoembryonic antigen (CEA) and cytokeratin 20 (CK20), can identify stage I and II patients with tumour cells in their lymph nodes that are undetectable by histopathology (Öberg et al, 2004; Ohlsson et al,

* Correspondence: Professor S Hammarström;

E-mail: sten.hammarstrom@climi.umu.se

Received 5 March 2012; revised 18 April 2012; accepted 24 April 2012; published online 14 June 2012
2006, 2012). We found, that real-time quantitative reverse transcriptase-PCR (qRT-PCR) analysis of mRNA for selected biomarkers is a superior method for tumour cell detection when used with a specific probe and an RNA copy standard (Öberg et al, 2004; Ohlsson et al, 2006, 2012). A problem with currently available biomarkers for CRC, including CEA, is that these are normal colonic tissue components and not tumour specific (Ohlsson et al, 2006, 2012). Thus, detection of CEA mRNA in regional lymph nodes indicates that tumour cells are of colonic origin but does not signify anything about tumour cell aggressiveness. Identification of a complementary marker to CEA reflecting also invasiveness would be of great interest.

Human tissue kallikrein-related peptidase 6 (KLK6) belongs to the human kallikrein gene family of serine proteases (Borgono and Diamandis, 2004). Many of these are dysregulated in human malignancies and have a role in cell growth regulation, angiogenesis, invasion and metastasis (Borgono and Diamandis, 2004; Talieri et al, 2009). Kallikrein-related peptidase 6 is expressed in ovarian cancer and appears to be an unfavourable progression biomarker in this malignancy (Anisowicz et al, 1996; Hoffman et al, 2002; Diamandis et al, 2003). Kallikrein-related peptidase 6 mRNA expression has been demonstrated in primary colorectal tumours (Yousef et al, 2004; Ogawa et al, 2005), and a smaller clinical study suggests that elevated KLK6 mRNA levels in the primary tumour might correlate to poor prognosis (Ogawa et al, 2005). In a recent study, it was suggested that, in the primary tumour, KLK6 mRNA together with 12 other biomarker mRNAs 
form a classifier for poor prognosis of CRC patients in stage II but not in stage III (Ågesen et al, 2012). At the protein level, KLK6 was detected in primary CRC tumours but there is a discrepancy between the two studies; whether it is detectable also in normal colon mucosa (Kim et al, 2011; Petraki et al, 2012).

In this study, we have focused on analysis of KLK6 mRNA expression in the lymph nodes of patients with CRC aiming at improved staging and prediction of outcome.

\section{MATERIALS AND METHODS}

\section{Patients}

Surgery for CRC was carried out in 166 patients (81 men, 85 women, median age 72 (range 42-90) years). In all, 13 of the tumours were located in the rectum and 153 in colon. Seven of the rectal cancer patients received $25 \mathrm{~Gy}$ of preoperative radiotherapy. A locally radical tumour resection was carried out in all patients. The tumour differentiation grade was poor, moderate and high in 11,145 and 10 tumours, respectively. Routine hematoxylin and eosin (H\&E) staining was performed on 2351 lymph nodes, giving a median of 13 (range 1-51) nodes per patient. According to the TNM classification, 30 patients were in stage I (T1-2N0M0), 74 in stage II (T3-4N0M0), 46 in stage III (anyTN1-2M0) and 16 in stage IV (anyTanyNM1). A total of 34 patients (4 in stage II, 19 in stage III and 11 in stage IV) received adjuvant chemotherapy after surgery. Follow-up was performed on 1 January 2011. No patient was lost at follow-up. The median follow-up time was 75 (range 33-147) months. Controls included 18 men and 5 women (median age 25 years (range 10-61)) undergoing surgery for ulcerative colitis (UC; $n=18)$, Crohn's colitis $(n=3)$, rectal prolapse $(n=1)$ and colon lipoma $(n=1)$.

Informed consent was obtained from the patients and in one case his parents. The Research Ethics Committee of the Medical Faculty, Umeå University, Sweden, approved the study.

\section{Lymph nodes}

Lymph nodes were retrieved from the resected specimens and bisected with separate, sterile knives. One half of each node was fixed in $10 \%$ buffered formalin for routine H\&E staining. The other half was snap frozen in liquid nitrogen and stored at $-70^{\circ} \mathrm{C}$ until RNA extraction (Ohlsson et al, 2009). From CRC patients, 503 lymph nodes $(91,253,107$ and 52 nodes from stage I, II, III and IV patients, respectively) were collected. A median of two (range 1-15) lymph nodes was obtained per patient. From control patients, 108 lymph nodes (82, 9, 13 and 4 nodes from UC, Crohn's colitis, colon lipoma and rectal prolapse patients, respectively) were collected.

\section{Primary and distant CRC tumour and normal colon tissue}

In all, 113 samples from 85 primary tumours were analysed (22 samples were from 16 stage I patients, 44 samples from 35 stage II patients, 41 samples from 25 stage III patients and 8 samples from 8 stage IV patients). Primary tumour stage distribution (pT1-pT4) was 2, 14, 55 and 13. The tumour differentiation grade was poor, moderate and high in 11, 71 and 3 tumours, respectively. The tumour samples, approximately $0.5 \times 0.5 \times 0.5 \mathrm{~cm}$ in size, were collected immediately after resection, snap-frozen and stored at $-70^{\circ} \mathrm{C}$ until RNA extraction. Six normal colon samples retrieved from the proximal or distal resection margin and two distant liver metastases samples were also collected and treated accordingly.

\section{Epithelial cells from colon tissue}

Colonic epithelial cells (ECs) were isolated from the normal colon mucosa at the resection margins as described (Fahlgren et al, 2003).

\section{Cell lines and peripheral blood mononuclear cells (PBMCs)}

Total RNA from the human cell lines LS174T, HT29, T84, HCT8 and $\mathrm{Caco} 2$ (all colon carcinomas), Jurkat and Molt-4 (T-cell lymphomas), B6 and KR4 (EBV-transformed B-cell lines), U266 (plasmacytoma cell line), U937 (monocyte-like cell line), K562 (erythroblastoid cell line), HL60 (granulocyte cell line) and FSU (fibroblast cell line) was analysed. Peripheral blood mononuclear cells were isolated from healthy adults by Ficoll-Isopaque gradient centrifugation, and RNA extracted directly and after polyclonal activation as described (Ohlsson et al, 2012).

\section{RNA preparation}

Total RNA was extracted using the acid guanidine phenol chloroform method as described (Ohlsson et al, 2012). The RNA concentration was measured in a NanoDrop ND-1000 Spectrophotometer (NanoDrop Technologies, Wilmington, DE, USA), and the integrity of the RNA was analysed in a 2100 Bioanalyzer using an RNA nano-assay (Agilent Technologies, Santa Clara, CA, USA).

\section{Microarray gene-expression analysis}

From each sample analysed, $400 \mathrm{ng}$ of total RNA was converted to biotinylated doublestranded cRNA according to the Illumina Totalprep RNA Amplification Kit (Ambion, Austin, TX, USA). The labelled cRNA samples were then hybridised on Sentrix HumanRef-8_V2 Expression Beadchips (Illumina, San Diego, CA, USA) incubated with streptavidin-Cy3 and scanned using the Illumina Beadstation GX (Illumina).

Results were analysed by using Illumina Beadstudio software (version 3.3) for direct hybridisation assays. Intensity data were normalised by Beadstudios cubic spline algorithm with subtracted background. Significant difference in expression was calculated using the Beadstudio software Error Model Illumina Custom with multiple testing corrections, using Benjamini and Hochberg false discovery rate (Reiner et al, 2003). Difference in gene expression was calculated as fold change, dividing the signal in individual CRC samples of interest over the average signal of controls.

\section{Real-time qRT-PCR}

Kallikrein-related peptidase $6 \mathrm{mRNA}$ occurs in different splice forms (Pampalakis et al, 2004). Before deciding which transcript(s) of KLK6 mRNA should be investigated in depth, we used three commercially available TaqMan Gene Expression Assays (Hs01100840_ml, Hs01105353_gl and Hs00160519_ml; Applied Biosystems, Foster City, CA, USA) to determine the expression levels of transcript A, transcript $B$ and all transcripts, respectively, in the test panel RNA samples described in Figure 1A. We found that the assay detecting all known transcripts and the assay detecting transcript $\mathrm{B}$ gave the same result with high mRNA expression in primary CRC and CRC cell lines and no expression in normal colon, immune cells or fibroblasts. The assay detecting transcript A showed the same tissue specificity; however, the expression levels were clearly lower (data not shown).

Consequently, a real time qRT-PCR assay with RNA copy standard detecting all known splice-forms of KLK6 mRNA was constructed by using the Taqman EZ technology (Applied Biosystems) as described (Fahlgren et al, 2003; Ohlsson et al, 2012). Primer and probe sequences were: forward primer $5^{\prime}$-CT TATCCATCCACTGTGGGTC-3', reverse primer $5^{\prime}$-TGGATCAC 


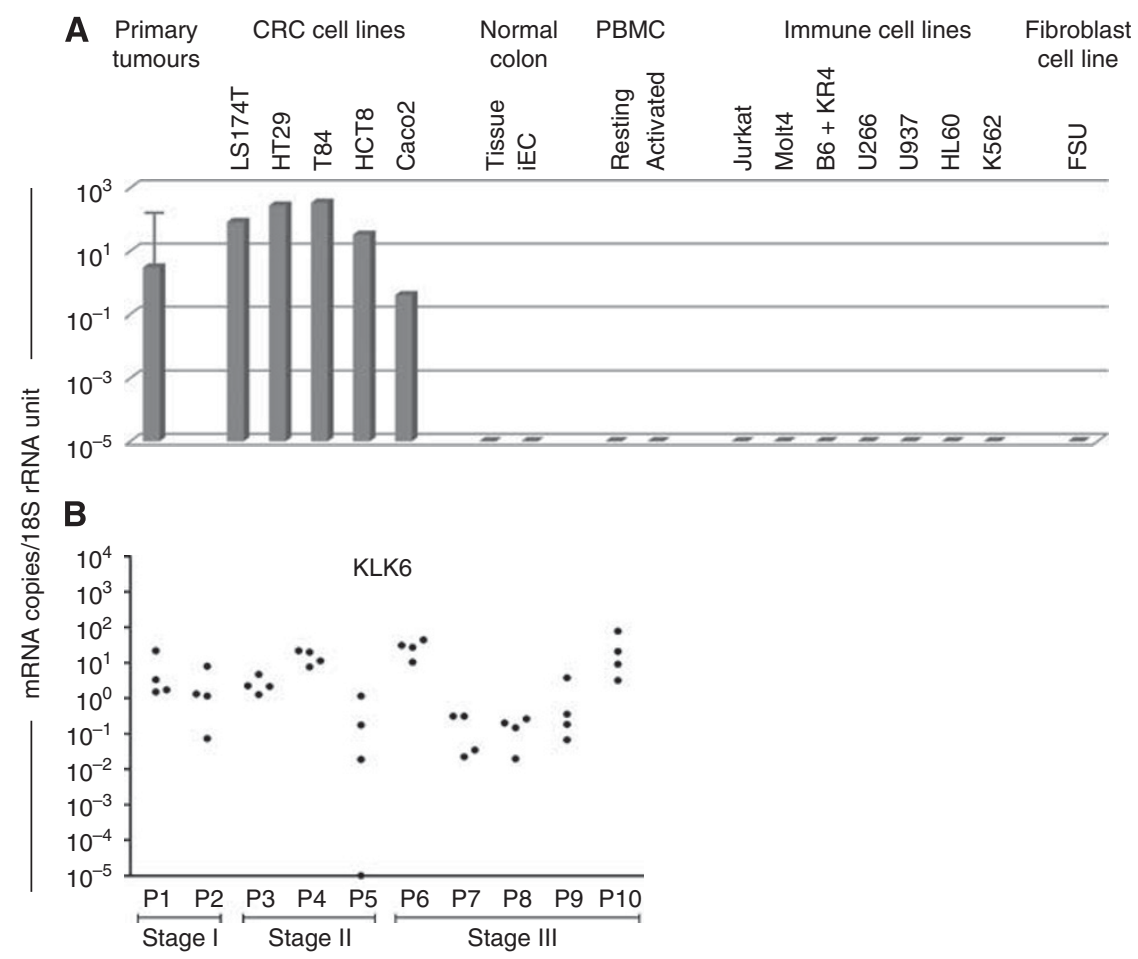

Figure I (A) Median KLK6 mRNA level in 85 primary CRC tumours (median: 3.0 mRNA copies/ I 8S rRNA unit; range: $0.01-137$ ), 6 normal colon tissue samples ( $<0.0000$ I mRNA copies/ I 8S rRNA unit), 6 normal colon EC samples ( $<0.0000$ I mRNA copies/ I 8 S rRNA unit) and mean of 3 determinations of the indicated cell lines and resting and activated human PBMC. KLK6 mRNA levels in LSI74T, HT29, T84, HCT8 and Caco2 cells were 82, $275,337,23$ and 0.4 mRNA copies/ I8S rRNA unit, respectively. KLK6 mRNA levels in PBMCs and all other cell lines: <0.0000 I mRNA copies/ /8S rRNA unit. (B) KLK6 mRNA levels at four different sites within individual primary tumours. Ten tumours were analysed, two from stage I patients (PI-P2), three from stage II patients (P3-P5) and five from stage III patients (P6-PIO).

AGCCCGGA-3' and probe 5'-CACTGCAAAAAACCGAATCTTC AGGTC-3'. The reporter dye was FAM and the quencher dye TAMRA. The RT-PCR profile was: $49^{\circ} \mathrm{C}$ for $2 \mathrm{~min}, 59^{\circ} \mathrm{C}$ for $30 \mathrm{~min}, 94^{\circ} \mathrm{C}$ for $5 \mathrm{~min}$ followed by 45 cycles of $93^{\circ} \mathrm{C}$ for $20 \mathrm{~s}$ and $61{ }^{\circ} \mathrm{C}$ for $1 \mathrm{~min}$. Emission from the released reporter dye was measured by the ABI Prism 7700 Sequence Detection System (Applied Biosystems). Serial dilutions of the RNA copy standard at concentrations from $10^{3}$ to $10^{8}$ copies per $\mu \mathrm{l}$ were included in each analysis. Concentrations in unknown samples were determined from the standard curve and expressed as copies of mRNA per $\mu$ l. All qRT-PCR analyses were carried out in triplicates. The reproducibility of the KLK6 assay was determined as described (Ohlsson et al, 2012). The coefficient of variation (CV) based on PCR ct-values was $1.0 \%$. The mean $\mathrm{CV}$ for estimation of mRNA content covering $10^{3}-10^{8}$ RNA copies per reaction was $26.7 \%$.

The concentration of $18 \mathrm{~S}$ rRNA was determined in each sample by real-time qRT-PCR for normalisation of mRNA levels as described (Bas et al, 2004; Ohlsson et al, 2012). Results are expressed as mRNA copies per unit of $18 \mathrm{~S}$ rRNA.

\section{Statistical analysis}

Correlation between biomarker mRNA levels was analysed using the non-parametric Spearman correlation coefficient. Differences in biomarker mRNA levels between two patient groups were analysed by two-tailed Mann-Whitney $U$ test. Descriptive values of mRNA levels are given as median and range or interquartile range (IQR) from the 25th to 75 th percentile.

Differences in disease-free survival and risk for recurrent disease after surgery between patient groups were calculated according to Kaplan-Meier survival model in combination with the log-rank test and univariate Cox regression analysis. Patients who died from causes other than CRC were considered as disease free. Descriptive values of risk and survival time are given as mean and $95 \%$ confidence interval (CI).

Correlations between mRNA levels, differences in mRNA levels, differences in survival time and hazard ratios with a $P$-value $<0.05$ were considered to be statistically significant. The software utilised was SPSS version 18 (IBM Corporation, Armonk, NY, USA).

\section{RESULTS}

To identify potential progression markers for CRC, we performed microarray analysis of gene-expression using seven different CRC RNA samples $(\mathrm{H} \& \mathrm{E}(+)$ lymph nodes of four stage III patients and the primary tumour from three of these) and seven control RNA samples (lymph nodes from two UC patients, one Crohns' colitis patient, one colon lipoma patient and three normal colon EC samples). Colorectal cancer samples were analysed individually relative to all control samples as one group. The microarray data were filtered by setting the inclusion criteria to a fold change of $\geqslant 5$, a statistical significance of $P<0.05$, and an intensity of $\geqslant 15$. Kallikrein-related peptidase 6 and 17 other genes fulfilled these criteria in all seven CRC samples (Supplementary Table 1).

The microarray data was verified by analysing a panel of RNA samples including primary CRC tumours, normal colon, CRC cell lines, PBMCs, immune cell lines and a fibroblast cell line for KLK6 mRNA levels using a qRT-PCR assay that is specific and detects all known splice-forms of KLK6 mRNA. Kallikrein-related peptidase 6 mRNA was expressed at relatively high levels in primary CRC tumours with a median value of $3.0 \mathrm{mRNA}$ copies/18S rRNA unit (IQR: 0.9-8.6). Interestingly, KLK6 was not expressed in normal colon or in any type of immune cell or in fibroblasts $(<0.00001$ 
mRNA copies/18S rRNA unit; Figure 1A). Of note, KLK6 mRNA was not expressed in activated PBMCs, in sharp contrast to the biomarker matrix metalloproteinase 7/matrilysin that was expressed at high levels in lymph nodes and activated PBMCs as compared with the resting PBMCs (Ohlsson et al, 2006). Analysis of samples obtained from different sites within the same tumour revealed that expression of KLK6 showed large intra-tumour heterogeneity. Kallikrein-related peptidase 6 levels could vary more than 100-fold between different sites in the same tumour (Figure 1B), while this was not the case for CEA levels in the same samples (Ohlsson et al, 2012). There was no correlation between KLK6 mRNA levels and pT-stages (data not shown).

Kallikrein-related peptidase $6 \mathrm{mRNA}$ was highly expressed in $2 / 2$ liver metastases (2.3 and $1.7 \mathrm{mRNA}$ copies/18S rRNA unit), but was not detected in normal liver.

\section{Lymph node KLK6 mRNA level increases with TNM stage}

In the following, each patient is represented by the node with the highest biomarker level. Figure 2A shows the KLK6 mRNA levels in the lymph nodes of $166 \mathrm{CRC}$ patients and 23 controls with benign disease. Kallikrein-related peptidase 6 mRNA was not detected in lymph nodes of controls $(<0.00001 \mathrm{mRNA}$ copies $/ 18 \mathrm{~S}$ rRNA unit) but in $20 \%$ of lymph nodes of stage I patients $(6 / 30)$, $11 \%$ of stage II patients $(8 / 74), 54 \%$ of stage III patients $(25 / 46)$ and $75 \%$ of stage IV patients (12/16). Kallikrein-related peptidase $6(+)$ lymph nodes had $\geqslant 0.0095$ mRNA copies/18S rRNA unit. The KLK6 levels in nodes of the few stage I and II patients who had a KLK6 $(+)$ lymph node were low (median 0.062 copies/18S rRNA unit) and clearly lower than most KLK6 $(+)$ nodes of stage III and IV patients (median 2.06 copies/18S rRNA unit; $P<0.0001$ ).

\section{Risk of recurrence increases with increasing lymph node KLK6 mRNA levels}

A total of 51 patients had died from CRC or were living with recurrent disease at follow-up (median 75 months, range 33-147), whereas 115 patients were apparently cured or had died of causes unrelated to CRC. Using Cox regression analysis, the risk of recurrence (hazard risk ratio) for the KLK6 $(+)$ and KLK6 $(-)$ populations was calculated. There was a 3.7-fold (95\% CI: 2.1-6.4) greater risk of recurrence of disease for KLK6 $(+)$ compared with KLK6( - ) patients (Table 1).

To evaluate whether the actual KLK6 mRNA level in lymph nodes is of importance for the risk of recurrence, we divided the patients into 10 groups of equal numbers according to the KLK6 mRNA levels. Up to the 70th percentile, patients had no detectable KLK6 mRNA. Using the cutoff values at the 70th, 80th and 90th percentiles hazard risk ratios were calculated (Table 1). Compared with KLK6 $(-)$ patients, KLK6(low) were not significantly different. Kallikrein-related peptidase 6(int) patients showed a 3.7-fold (95\% CI: 1.7-8.0) increased risk compared with KLK6( - ) patients and KLK6(high) an 6.5-fold (95\% CI: 3.2-13.3) increased risk, both found to be statistically highly significant (Table 1).

\section{Survival time after surgery decreases with increasing lymph node KLK6 mRNA levels}

The cumulative survival of lymph node KLK6 $(+)$ and lymph node KLK6 $(-)$ patients estimated by Kaplan-Meier analyses is shown in Figure 3A and Table 1. The mean survival time for KLK6(-) patients was 117 months and 54 months for KLK6(+) patients, giving a highly significant difference in survival of 63 months. Kallikrein-related peptidase 6(low), KLK6(int) and KLK6(high) patients showed 49, 63 and 76 months shorter mean survival times than KLK6 $(-)$ patients, respectively (Figure 3B and Table 1). Thus, short survival time correlates with high KLK6 mRNA levels in resected lymph nodes.
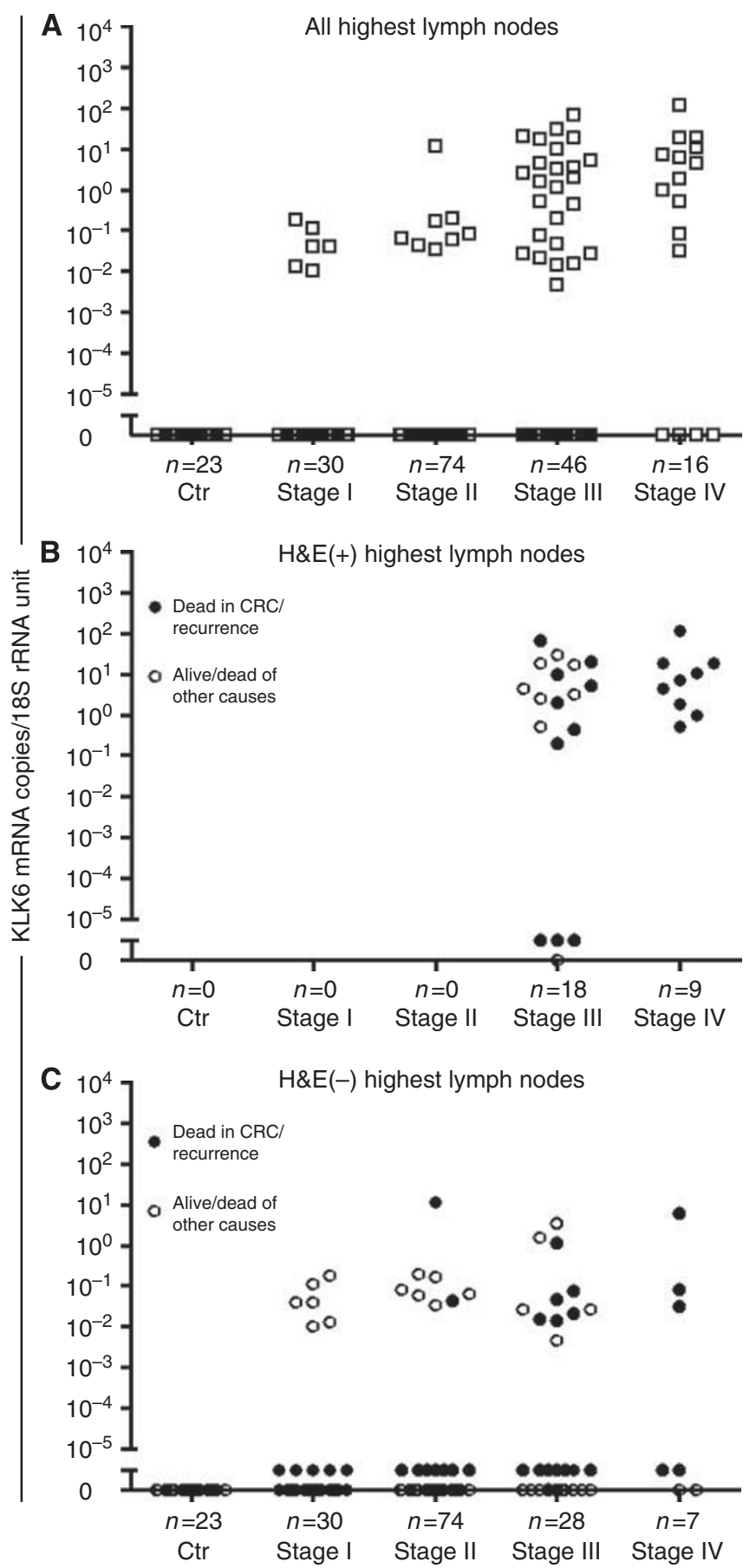

Figure 2 KLK6 mRNA levels in lymph nodes of stage I to IV CRC patients and control patients (Ctr). Each of the 166 CRC patients and 23 control patients is represented by the lymph node with the highest KLK6 level. (A) All patients, (B) patients with at least one H\&E $(+)$ lymph node available for mRNA analysis $(\mathbf{C})$ patients with only $\operatorname{H\& E}(-)$ lymph nodes available for mRNA analysis. Filled circles represent patients who have died in CRC or are living with recurrent disease. Open circles represent living, disease-free patients and patients who have died from other causes than CRC. For clarity dots indicating KLK6 ( - ) patients who have died from CRC are positioned slightly above those who are alive in $(\mathbf{B})$ and $(\mathbf{C})$.

This result was underscored when prognosis was analysed by plotting the proportion of patients who had died from CRC or were living with recurrent disease against years after surgery (Figure 3C). Colorectal cancer patients with high lymph node 
Table I Comparative analysis of average survival time after surgery and risk for recurrence of disease of CRC patients with KLK6 $(+)$ or KLK6 $(-)$ lymph nodes

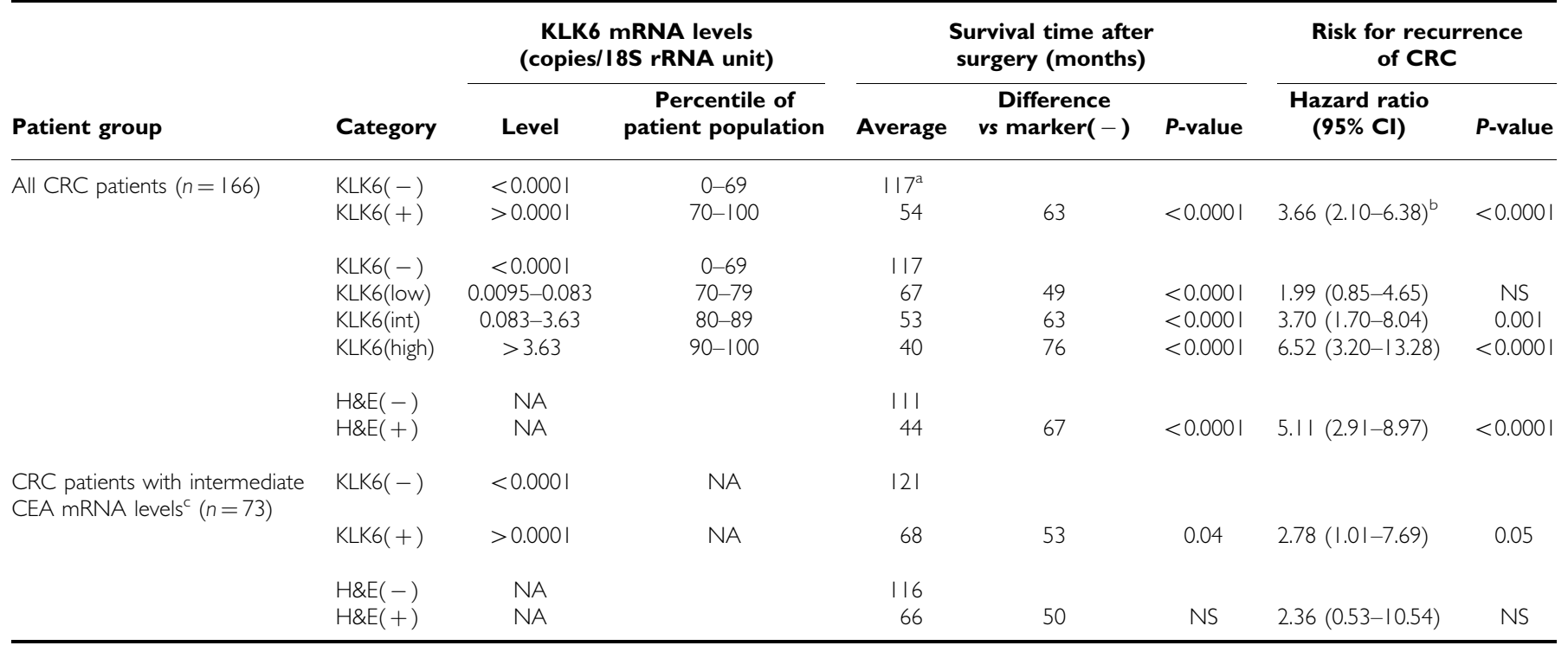

Abbreviations: $\mathrm{Cl}=$ confidence interval; $\mathrm{NA}=$ not applicable; $\mathrm{NS}=$ not significant $(P$-value $>0.05)$. ${ }^{a}$ Mean survival time after surgery as calculated by cumulative survival analysis

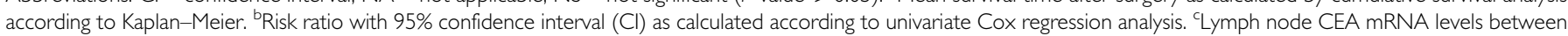
0.013 and 3.67 mRNA copies/I8S rRNA unit, that is, above the level in lymph nodes of controls and below the clinical cutoff for CEA mRNA (Ohlsson et al, 20I2).
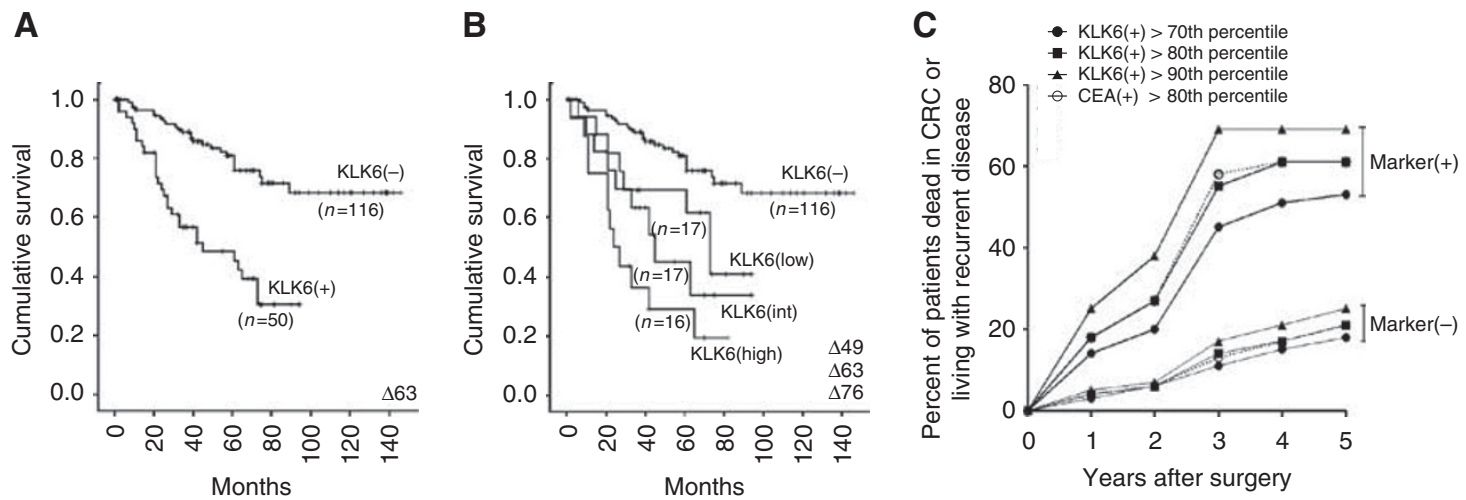

Figure 3 Cumulative survival curves according to Kaplan-Meier for CRC patients with $(\mathbf{A}) \mathrm{KLK} 6(+)$ lymph nodes, (B) KLK6(high), KLK6(int), and KLK6(low) lymph nodes, and (A, B) KLK6 (-) lymph nodes. The difference in average survival time between the KLK6(+), KLK6(high), KLK6(int) and KLK6 (low) versus KLK6 ( - ) patients is given as a delta value in months for each patient group. (C) Proportion of CRC patients with recurrent disease in marker positive (marker $(+)$ ) and marker negative (marker $(-)$ ) groups as a function of time after surgery. (-) Patients with a KLK6 mRNA level above the 70th percentile, that is, 0.0095 mRNA copies/I8S rRNA unit; ( $)$ Patients with a KLK6 mRNA level above the 80th percentile, that is, 0.00083 mRNA copies/I8S rRNA unit; $(\boldsymbol{\Delta})$ Patients with a KLK6 mRNA level above the 90th percentile, that is, 3.63 mRNA copies/ I8S rRNA unit; (O and dotted line) Patients with a CEA mRNA level above the clinical cutoff at the 80th percentile, that is, 3.67 mRNA copies/ $8 \mathrm{~S}$ rRNA unit. $n=$ numbers of patients in the respective group.

KLK6 mRNA levels died earlier than patients with intermediate and in particular with low KLK6 mRNA levels. The differences were most pronounced within the first 2 years. At this time, there was no difference in the proportion of patients who had died from recurrent disease in the respective marker negative patient group.

\section{Kallikrein-related peptidase 6 mRNA identifies stage I and II patients with lymph node occult disease}

Stage I and II patients are by definition $\operatorname{H\& E}(-)$, thus presumed to have no tumour cells in their lymph nodes. In all, 14 of 104 stage I and II patients were KLK6 $(+)$. Interestingly, two of the
KLK6 $(+)$ patients had died from recurrent CRC at follow-up (Figure 2C). In addition, 2 of the H\&E( -$)$ KLK6 $(+)$ patients had died from unrelated diseases, whereas 10 were alive without apparent recurrence. Thus, KLK6 mRNA analysis detects positive cells in $13 \%$ of the patients who were negative in H\&E staining and some of these succumbed from CRC.

Altogether, 14 stage I and II patients had died from CRC or were living with tumour recurrence (Figure 2C). All but two were $\mathrm{H} \& \mathrm{E}(-) \mathrm{KLK} 6(-)$ and therefore missed by both types of lymph node analysis. Possibly, they were missed because tumour cells spread by the hematogenous route or because too few lymph nodes were analysed. 

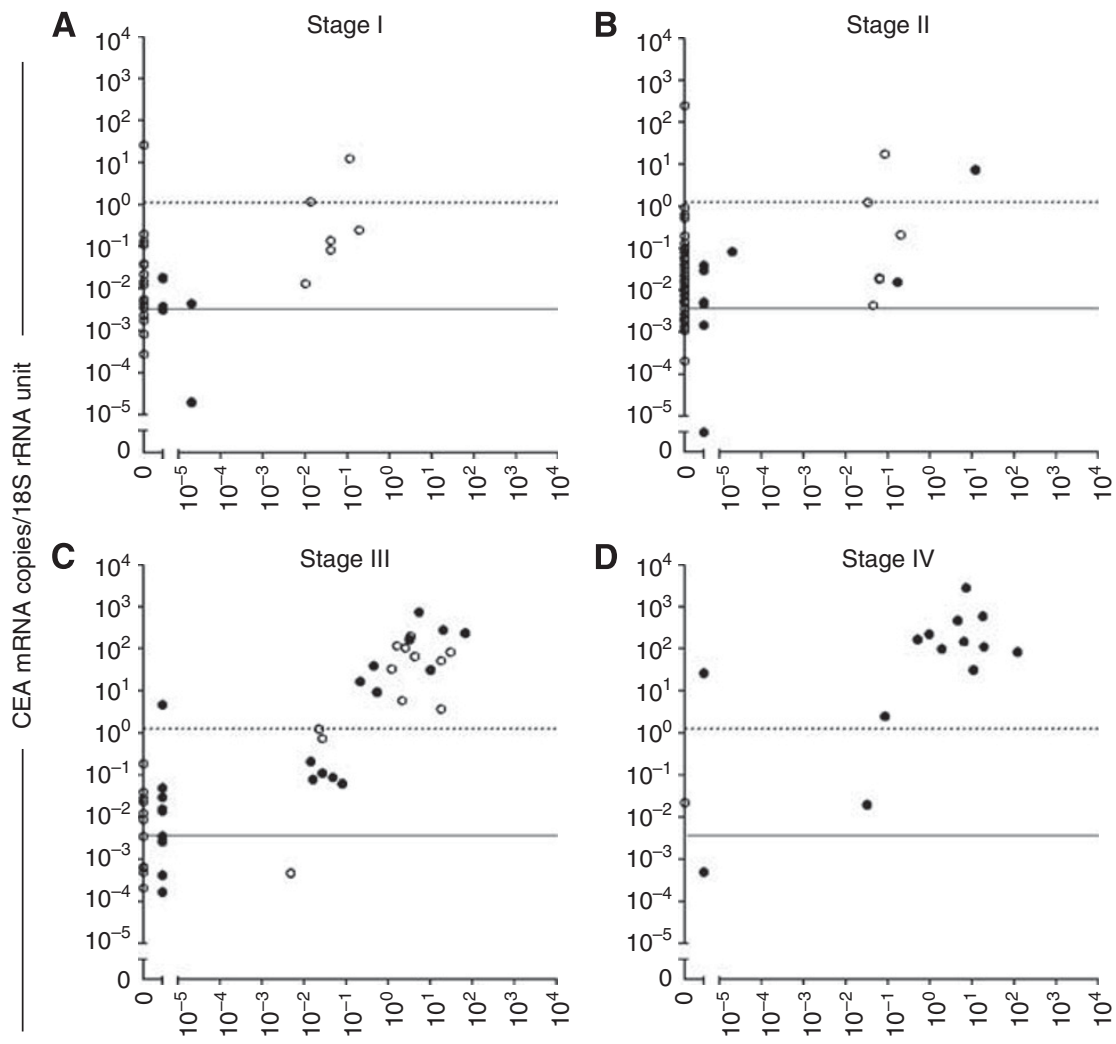

KLK6 mRNA copies/18S rRNA unit
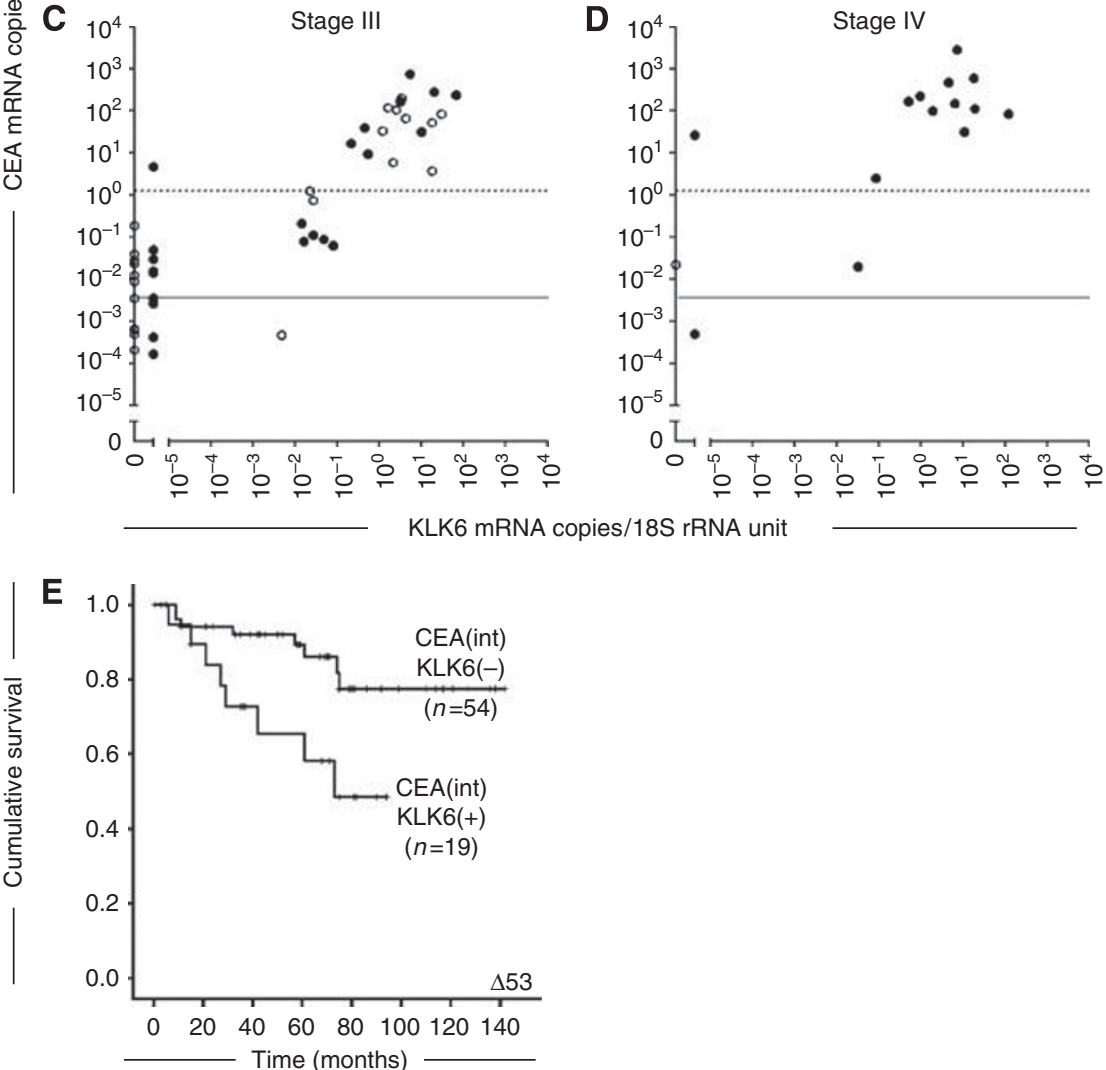
group comprised patients from whom only $\mathrm{H} \& \mathrm{E}(-)$ nodes were available for mRNA analysis (Figure $2 \mathrm{~B}$ and $\mathrm{C}$ ). In the first group, KLK6 mRNA identified 16 of the 19 patients who had died from $\mathrm{CRC}$ or were living with recurrent disease (Figure 2B). In the second group, 18 stage III and IV patients had died from CRC. Of these, nine patients were KLK6 $(+)$ (Figure 2C). Thus, $50 \%$ of $\mathrm{H} \& \mathrm{E}(-)$ nodes from stage III and IV patients that succumbed from CRC were identified by KLK6 mRNA. On the other hand, 4 of the $27(14 \%) \mathrm{H} \& \mathrm{E}(+)$ nodes from stage III and IV patients were KLK6 $(-)$ and 3 of them had died from CRC (Figure 2B). Complementary KLK6 mRNA analysis identifies aggressive
tumour cells in lymph nodes with few occult tumour cells

Previous comparative studies demonstrated that CEA mRNA performs better for the detection of disseminated tumour cells in the lymph nodes of patients with CRC than 10 other investigated biomarker mRNAs (Ohlsson et al, 2006, 2012). Analysing CEA mRNA, there is a group of patients with intermediate levels (CEA(int)), that is, higher than controls but below the clinical cutoff established by Cox regression analysis (Ohlsson et al, 2012). We explored whether KLK6 would be useful as a complementary biomarker in detecting aggressive tumour cells in CEA(int) patients.

Figures 4A-D show the results of KLK6 - and CEA mRNA analyses of lymph nodes from CRC patients of all tumour stages. In the CEA(int) group of patients, one stage II (Figure 4B), five stage III (Figure 4C) and two stage IV (Figure 4D) patients had died from CRC had KLK6 $(+)$ nodes. Thus, KLK6 $(+)$ identifies a subgroup of patients, who on the basis of CEA only, would not have been classified as patients at risk for tumour recurrence. On the other hand, there were eight additional CEA(int) KLK6(-) patients who had died from CRC who would have been undetected by both biomarkers.

Carcinoembryonic antigen(int) patients were divided into KLK6 $(+)$ and KLK6 $(-)$ groups and analysed for the risk of recurrence and cumulative survival. Kallikrein-related peptidase $6(+)$ was an indicator for poor prognosis with a hazard ratio of 2.8 and on average 53 months shorter survival (Table 1 and Figure 4E). Thus, KLK6 positivity even in lymph nodes with few tumour cells is a sign of tumour aggressiveness.

\section{DISCUSSION}

The aim of this study was to identify a tumour progression marker for CRC that could be used alone or complementary to CEA mRNA analysis identifying high-risk patients with poor prognosis and in need of adjuvant chemotherapy, but also identifying patients with good prognosis that should be spared from additional therapy.

In this study, we identified KLK6 mRNA as a most promising progression biomarker for CRC. Kallikrein-related peptidase 6 mRNA was expressed in all analysed primary tumours, but was not expressed in normal colon. This finding is of great interest as all other CRC biomarkers studied so far have shown high expression levels in normal adult colon (Ohlsson et al, 2006, 2012). Thus, KLK6 mRNA expression in colon appears to be a sign of malignant transformation. Our findings are in line with previous studies on KLK6 mRNA and protein in primary CRC tumour and normal colon, although low levels of KLK6 mRNA and protein was reported in a few samples of normal colon tissue (Ogawa et al, 2005; Kim et al, 2011). One recent study reports KLK6 protein in normal colon but at the same time these authors report poor prognosis for CRC patients with intense KLK6 staining of primary tumours (Petraki et al, 2012). We believe that the minor discrepancy at the mRNA level and the discrepancies at the protein level relate to the specificities of the mRNA assays and antibody reagents, respectively.

To determine whether KLK6 is a useful prognostic marker for survival, we focused on studies of the mesenteric lymph nodes because the mere presence of tumour cells, particularly in several lymph nodes, is in itself a sign of poor prognosis. That lymph node analysis is to be preferred rather than analysis of primary tumour is supported by a recent study, which suggests that KLK6 mRNA analysis of primary tumour is useful only in stage II patients (Ågesen et al, 2012). Kallikrein-related peptidase 6 mRNA analysis was also compared with CEA mRNA analysis and H\&E staining of the same lymph nodes. The following tentative conclusions could be drawn: (1) KLK6 $(+)$ lymph nodes were always CEA(+). Thus, KLK6 mRNA only occurred in lymph nodes with cells derived from the epithelium of colon or rectum. (2) Kallikrein-related peptidase 6 mRNA was expressed in only 10-20\% of lymph nodes from stage I and II patients but in $53 \%$ and $75 \%$ of lymph nodes from stage III and IV patients, respectively. Moreover, the mRNA levels in the KLK6 $(+)$ stage I and II patients were 30 times lower than in stage III and IV patients. (3) The hazard ratio for the risk of recurrence was 3.7 times higher in the KLK6 $(+)$ group, as compared to the KLK6 $(-)$ group, and the difference in the mean survival time was 63 months between groups. (4) The actual KLK6 mRNA level in the lymph node was directly related to the risk of recurrence. Thus, patients with the highest KLK6 levels had the highest hazard risk ratio and the shortest survival time. (5) Patients with high KLK6 mRNA levels in lymph nodes tended to succumb from recurrence within 3 years after surgery. (6) A group of stage III and IV patients with intermediate CEA mRNA levels expressed high KLK6 mRNA. These patients had CEA values below the clinical cutoff and would therefore, on the basis of CEA alone, not have been considered to be at risk for recurrent disease and possibly undertreated (Ohlsson et al, 2012). Kallikrein-related peptidase $6 \mathrm{mRNA}$ identified more than $60 \%$ of the patients in this group who died from recurrent CRC. (7) Kallikrein-related peptidase 6 mRNA was highly expressed in liver metastases. We conclude that KLK6 mRNA is a most valuable progression marker in CRC.

The functional properties of KLK6 support the notion that it is a progression marker. As a secreted serine protease KLK6 has the potential of being instrumental in the metastatic process (Borgono and Diamandis, 2004). Thus, KLK6 $(+)$ tumour cells may have a greater chance of escaping the primary site and to metastasise to distant sites than KLK6 $(-)$ tumour cells. Kallikrein-related peptidase 6 can degrade basic constituents of the extracellular matrix and the basement membrane such as fibrinogen, collagen I and IV, fibronectin, vitronectin and laminin (Magklara et al, 2003). Activating K-RAS mutations are common in CRC, occurring in about $30 \%$ of all CRC cases. Activation of K-RAS involves increased proliferation, evasion of apoptosis and increased migration and invasion. One important explanation for KLK6 mRNA being expressed in CRC is that KLK6 functions as a mediator of K-RAS-dependent migration (Henkhaus et al, 2008). Moreover, KLK6 reduces E-cadherin levels in the cell membrane, probably by indirectly affecting ectodomain shedding leading to reduced cell-cell adhesion (Klucky et al, 2007).

Combined analysis of CEA and KLK6 mRNAs in lymph nodes show great potential in identifying patients at high risk of tumour recurrence. We believe that the combined analysis of CEA and KLK6 mRNA in lymph nodes will become a powerful prognostic tool in CRC. This can easily be achieved by utilising qRT-PCR amendable to automation.

\section{ACKNOWLEDGEMENTS}

We sincerely thank Malin Sund MD, PhD of the Department of Surgery and Perioperative Sciences, Umeå University, for liver metastases and normal liver samples.

Supplementary Information accompanies the paper on British Journal of Cancer website (http://www.nature.com/bjc) 


\section{REFERENCES}

Anisowicz A, Sotiropoulou G, Stenman G, Mok SC, Sager R (1996) A novel protease homolog differentially expressed in breast and ovarian cancer. Mol Med 2: 624-636

Ågesen TH, Sveen A, Merok MA, Lind GE, Nesbakken A, Skotheim RI, Lothe RA (2012) ColoGuideEx: a rubust gene classifier specific for stage II colorectal cancer prognosis. Gut; e-pub ahead of print 2 January 2012; doi:10.1136/gutjnl-2011-301179

Bas A, Forsberg G, Hammarström S, Hammarström ML (2004) Utility of the housekeeping genes $18 \mathrm{~S}$ rRNA, $\beta$-actin and glyceraldehyde-3-phosphatedehydrogenase for normalization in real-time quantitative reverse transcriptase-polymerase chain reaction analysis of gene expression in human T lymphocytes. Scand J Immunol 59: 566-573

Bilchik AJ, Hoon DS, Saha S, Turner RR, Wiese D, DiNome M, Koyanagi K, McCarter M, Shen P, Iddings D, Chen SL, Gonzalez M, Elashoff D, Morton DL (2007) Prognostic impact of micrometastases in colon cancer: interim result of a prospective multicenter trial. Ann Surg 246: 568-575

Borgono C, Diamandis E. The emerging roles of human tissue kallikreins in cancer (2004) Nat Rev Cancer 4: 876-890

Chang GJ, Rodriguez-Bigas MA, Skibber JM, Moyer VA (2007) Lymph node evaluation and survival after curative resection of colon cancer: systematic review. J Natl Cancer Inst 99: 433-441

Diamandis EP, Scorilas A, Fracchioli S, Van Gramberen M, De Bruijn H, Henrik A, Soosaipillai A, Grass L, Yousef GM, Stenman UH, Massobrio M, Van Der Zee AG, Vergote I, Katsaros D (2003) Human kallikrein 6 (hK6): a new potential serum biomarker for diagnosis and prognosis of ovarian carcinoma. J Clin Oncol 21: 1035-1043

Fahlgren A, Hammarström S, Danielsson Å, Hammarström ML (2003) Increased expression of antimicrobial peptides and lysozyme in colonic epithelial cells of patients with ulcerative colitis. Clin Exp Immunol 131: 90-101

Henkhaus RS, Gerner EW, Ignatenko NA (2008) Kallikrein 6 is a mediator of K-RAS-dependent migration of colon carcinoma cells. Biol Chem 389: 757-764

Hoffman BR, Katsaros D, Scorilas A, Diamandis P, Fracchioli S, Rigault de la Longrais IA, Colgan T, Puopolo M, Giardina G, Massobrio M, Diamandis EP (2002) Immunofluorometric quantitation and histochemical localisation of kallikrein 6 protein in ovarian cancer tissue: a new independent unfavourable prognostic biomarker. $\mathrm{Br}$ J Cancer 87: 763-771

Iddings D, Bilchik A (2007) The biologic significance of micrometastatic disease and sentinel lymph node technology on colorectal cancer. J Surg Oncol 96: 671-677

Kim JT, Song EY, Chung KS, Kang MA, Kim JW, Kim SJ, Yeom YI, Kim JH, Kim KH, Lee HG (2011) Up-regulation and clinical significance of serine protease kallikrein 6 in colon cancer. Cancer 117: 2608-2619

Klucky B, Mueller R, Vogt I, Teurich S, Hartenstein B, Breuhahn K, Flechtenmacher C, Angel P, Hess J (2007) Kallikrein 6 induces E-cadherin shedding and promotes cell proliferation, migration, and invasion. Cancer Res 67: 8198-8206

Magklara A, Mellati AA, Wasney GA, Little SP, Sotiropoulou G, Becker GW, Diamandis EP (2003) Characterization of the enzymatic activity of human kallikrein 6: autoactivation, substrate specificity, and regulation by inhibitors. Biochem Biophys Res Commun 307: 948-955

Nicastri DG, Doucette JT, Godfrey TE, Hughes SJ (2007) Is occult lymph node disease in colorectal cancer patients clinically significant? A review of the relevant literature. J Mol Diagn 9: 563-571

Öberg ÅN, Lindmark GE, Israelsson AC, Hammarström SG, Hammarström ML (2004) Detection of occult tumor cells in lymph nodes of colorectal cancer patients using real-time quantitative RT-PCR for CEA and CK20 mRNAs. Int I Cancer 111: 101-110

Ogawa K, Utsunomiya T, Mimori K, Tanaka F, Inoue H, Nagahara H, Murayama S, Mori M (2005) Clinical significance of human kallikrein gene 6 messenger RNA expression in colorectal cancer. Clin Cancer Res 11: 2889-2893

Ohlsson L, Hammarström ML, Israelsson A, Näslund L, Öberg Å, Lindmark G, Hammarström S (2006) Biomarker selection for detection of occult tumor cells in lymph nodes of colorectal cancer patients using real-time quantitative RT-PCR. Br J Cancer 95: 218-225

Ohlsson L, Israelsson A, Öberg A, Hammarström ML, Lindmark G, Hammarström S (2009) Detection of tumor cells in lymph nodes of colon cancer patients using real-time quantitative reverse transcription-polymerase chain reaction. In Methods of Cancer Diagnosis, Therapy, and Prognosis, Hayat MA (ed) pp 257-268. Springer: New York

Ohlsson L, Israelsson A, Öberg $\AA$, Palmqvist R, Stenlund H, Hammarström ML, Hammarström S, Lindmark G (2012) Lymph node CEA and MUC2 mRNA as useful predictors of outcome in colorectal cancer. Int J Cancer 130: $1833-1843$

Pampalakis G, Kurlender L, Diamandis EP, Sotiropoulou G (2004) Cloning and characterization of novel isoforms of the human kallikrein 6 gene. Biochem Biophys Res Commun 320: 54-61

Petraki C, Dubinski W, Scorilas A, Saleh C, Pasic MD, Komborozos V, Khalil B, Gabril MY, Streutker C, Diamandis EP, Yousef GM (2012) Evaluation and prognostic significance of human tissue kallikreinrelated peptidase 6 (KLK6) in colorectal cancer. Pathol Res Pract 208: 104-108

Reiner A, Yekutieli D, Benjamini Y (2003) Identifying differentially expressed genes using false discovery rate controlling procedures. Bioinformatics 19: 368-375

Talieri M, Li L, Zheng Y, Alexopoulou DK, Soosaipillai A, Scorilas A, Xynopoulos D, Diamandis EP (2009) The use of kallikrein-related peptidases as adjuvant prognostic markers in colorectal cancer. $\mathrm{Br} J$ Cancer 100: 1659-1665

Yousef GM, Borgoño CA, Popalis C, Yacoub GM, Polymeris ME, Soosaipillai A, Diamandis EP (2004) In-silico analysis of kallikrein gene expression in pancreatic and colon cancers. Anticancer Res 24: 43-51

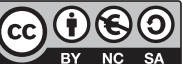

This work is licensed under the Creative Commons Attribution-NonCommercial-Share Alike 3.0 Unported License. To view a copy of this license, visit http://creativecommons. org/licenses/by-nc-sa/3.0/ 